\title{
Endoscopic Ultrasound Real-Time Elastography in Liver Disease
}

\author{
Jeong Eun Song, Dong Wook Lee and Eun Young Kim \\ Division of Gastroenterology, Department of Internal Medicine, Catholic University of Daegu School of Medicine, Daegu, Korea
}

See "A Prospective Blinded Study of Endoscopic Ultrasound Elastography in Liver Disease: Towards a Virtual Biopsy" by Allison R. Schulman, Ming V. Lin, Anna Rutherford, et al., on page 181-185.

In patients with chronic liver disease (CLD), persistent inflammation and tissue injury lead to liver fibrosis. The degree of hepatic fibrosis should be evaluated accurately for therapeutic decision-making and estimation of prognosis. ${ }^{1}$ Liver biopsy is still recommended as the gold standard for assessment of the degree of hepatic fibrosis ${ }^{2}$ but it is not suitable for serial monitoring of the dynamic changes in fibrosis because of its invasiveness. $^{3}$

Recently, non-invasive methods to assess the extent of hepatic fibrosis have emerged as substitutes for liver biopsy. Among these, ultrasound elastography techniques to assess liver stiffness related to hepatic fibrosis have been introduced, including transient elastography (TE), real-time elastography (RTE), acoustic radiation force impulse imaging (ARFI), and shear wave elastography (SWE). ${ }^{4}$ Although TE, ARFI, and SWE provide quantitative values of liver stiffness by measuring the speed of shear waves, the computation processes and results are different among these modalities. A shear wave is produced by an acoustic pulse or a vibration, and spreads through the tissue examined. Strain elastography is an operator-dependent procedure and can be influenced by surrounding tissues or ascites. Different from other ultrasound elastography techniques,

Received: March 3, 2018 Revised: March 19, 2018

Accepted: March 19, 2018

Correspondence: Eun Young Kim

Department of Internal Medicine, Catholic University of Daegu School of Medicine, 33 Duryugongwon-ro 17-gil, Nam-gu, Daegu 42472, Korea

Tel: +82-53-650-4092, Fax: +82-53-624-3281, E-mail: kimey@cu.ac.kr ORCID: https://orcid.org/0000-0003-3965-9964

(c) This is an Open Access article distributed under the terms of the Creative Commons Attribution Non-Commercial License (http://creativecommons.org/ licenses/by-nc/3.0) which permits unrestricted non-commercial use, distribution, and reproduction in any medium, provided the original work is properly cited.
RTE can measure heartbeat-induced strain and thus provide relatively objective qualitative (color-coded strain map) and semiquantitative results (scores such as liver fibrosis index [LFI], elasticity index, and elastic ratio). ${ }^{5}$

Among the above-mentioned ultrasound-based techniques, TE (FibroScan ${ }^{\mathrm{TM}}$ ) was introduced first and is now used globally in clinical settings. Its usefulness was reported in many previous studies that demonstrated reliable diagnostic accuracy for the evaluation of fibrosis in CLDs with various etiologies. ${ }^{6,7}$ The limitation of TE is the difficulty of measuring liver stiffness in patients with high body mass index (BMI), narrow intercostal spaces, severe hepatic atrophy, and ascites. ${ }^{8}$ In a prior study, BMI $>28 \mathrm{~kg} / \mathrm{m}^{2}$ was significantly associated with failure of liver stiffness measurement using TE. ${ }^{9}$ Transabdominal RTE is a more sensitive method of hepatic fibrosis measurement than TE. Compared to TE, RTE sometimes has shown slight inferior diagnostic accuracy but is known to have few or no limitations. Among many previous studies, Koizumi et al. ${ }^{3}$ reported that skinfold thickness and BMI did not significantly affect the elastic ratio determined when examiners measured liver stiffness at four sites through intercostal body surface using RTE. Another more recent study by Marques et al., ${ }^{10}$ however, showed that abdominal wall thickness of $\geq 23 \mathrm{~mm}$ was significantly associated with unsuccessful measurement of LFI using RTE.

While many studies to date have paid attention to transabdominal RTE, the article in this issue of Clinical Endoscopy by Schulman et al. ${ }^{11}$ introduced a pilot study of endoscopic ultrasound (EUS) RTE for distinguishing normal liver, fatty liver, and cirrhosis in patients with CLD. It is a reasonable idea that EUS RTE may be more sensitive than transabdominal RTE in assessing the stage of liver fibrosis because of the shorter 
penetration depth in the EUS approach than in the transabdominal approach (thin gastric wall vs. thick abdominal wall). The study evaluated 50 prospectively enrolled patients who underwent EUS RTE and abdominal imaging prior to EUS. The patients were divided into normal liver $(n=26)$, fatty liver $(n=16)$, and cirrhosis groups $(n=8)$. LFI computed from the EUS RTE was statistically different among the normal liver, fatty liver, and cirrhosis groups. The cirrhotic group had a significantly higher mean LFI than the fatty liver (3.2 vs. 1.7, $p<0.001$ ) and normal groups ( 3.2 vs. $0.8, p<0.001)$. When applying a previously reported LFI cut-off value, ${ }^{12}$ the area under the receiver-operating characteristic curve for LFI in predicting cirrhosis on imaging was 0.865 .

When evaluating the results of this study, some limitations should be considered. First, it is not clear what kind of abdominal imaging was used to distinguish normal liver, fatty liver, and cirrhosis. Furthermore, the definitions of normal liver, fatty liver, and cirrhosis were not described in detail. Abdominal ultrasonography is the most common first-line imaging study for patients with CLD. However, ultrasonography does not perform well in obese patients ${ }^{13}$ and may miss the diagnosis of fatty liver if steatosis is $\leq 30 \%{ }^{14}$ In this study, the mean BMIs of the patients were relatively high at $29.6 \mathrm{~kg} / \mathrm{m}^{2}$, $30.8 \mathrm{~kg} / \mathrm{m}^{2}$ and $28.3 \mathrm{~kg} / \mathrm{m}^{2}$ in the normal, fatty liver, and liver cirrhosis groups, respectively. If abdominal ultrasonography is used for imaging, mild fatty liver may be misdiagnosed as normal liver. Second, liver biopsy was performed not in all patients; thus, no data were available for fibrosis stage in this study. Although liver biopsy has several limitations, it is still considered as the gold standard assessment for liver fibrosis stage. Many previous studies that used noninvasive methods validated their ability to identify significant fibrosis and cirrhosis in comparison with liver biopsy. ${ }^{3,610,12}$ Those studies suggested a cutoff value for each fibrosis stage on the basis of liver biopsy results. Fatty liver is comprised of a broad spectrum of characteristics, including isolated steatosis, steatohepatitis, advanced fibrosis, and cirrhosis. The role of liver biopsy is to establish a diagnosis, assess fibrosis stage, and correlate histological lesions with potential clinical outcomes. ${ }^{15}$ Without performing liver biopsy, broad-spectrum characteristics could not be interrogated properly and taken into account. Thus, the LFI of the fatty liver group could not be generalized to every patient with fatty liver. Another significant limitation of this study was its small sample size $(n=50)$, especially in the cirrhosis group ( $n=8)$, which comprised $16 \%$ of all recruited patients. This limits the strength to differentiate the three groups and predict cirrhosis on imaging using the LFI.

Although this study has some limitations, it demonstrates that EUS RTE might be a potentially effective method for noninvasive assessment of liver fibrosis, especially in obese pa- tients. However, invasiveness of EUS RTE compared to transabdominal RTE and sedation needed for EUS are drawbacks and obstacles for implementation of EUS RTE. Increased sedation requirement in patients with obesity may raise the risk of adverse events such as obstructive sleep apnea. ${ }^{16}$ Further studies are required to compare the efficacy of EUS RTE with that of transabdominal RTE and liver biopsy to determine the cut-off value for EUS RTE according to fibrosis stage and to determine the safety of EUS RTE.

\section{Conflicts of Interest}

The authors have no financial conflicts of interest.

\section{REFERENCES}

1. European Association for the Study of the Liver. EASL 2017 clinical practice guidelines on the management of hepatitis B virus infection. J Hepatol 2017;67:370-398.

2. Bravo AA, Sheth SG, Chopra S. Liver biopsy. N Engl J Med 2001;344:495500 .

3. Koizumi Y, Hirooka M, Kisaka Y, et al. Liver fibrosis in patients with chronic hepatitis C: noninvasive diagnosis by means of real-time tissue elastography--establishment of the method for measurement. Radiology 2011;258:610-617.

4. Sporea I, Sirli RL. Hepatic elastography for the assessment of liver fibrosis--present and future. Ultraschall Med 2012;33:550-558.

5. Frey H. [Realtime elastography. A new ultrasound procedure for the reconstruction of tissue elasticity]. Radiologe 2003;43:850-855.

6. Kim DY, Kim SU, Ahn SH, et al. Usefulness of FibroScan for detection of early compensated liver cirrhosis in chronic hepatitis B. Dig Dis Sci 2009;54:1758-1763.

7. Kim SU, Park JY, Kim DY, et al. Non-invasive assessment of changes in liver fibrosis via liver stiffness measurement in patients with chronic hepatitis B: impact of antiviral treatment on fibrosis regression. Hepatol Int 2010;4:673-680.

8. Castera L. Transient elastography and other noninvasive tests to assess hepatic fibrosis in patients with viral hepatitis. J Viral Hepat 2009;16:300-314.

9. Foucher J, Castéra L, Bernard PH, et al. Prevalence and factors associated with failure of liver stiffness measurement using FibroScan in a prospective study of 2114 examinations. Eur J Gastroenterol Hepatol 2006;18:411-412.

10. Marques S, Carmo J, Túlio MA, Bispo M, Matos L, Chagas C. Diagnostic performance of real-time elastography in the assessment of advanced fibrosis in chronic hepatitis C. GE Port J Gastroenterol 2016;23:13-18.

11. Schulman AR, Lin MV, Rutherford A, Chan WW, Ryou M. A prospective blinded study of endoscopic ultrasound elastography in liver disease: towards a virtual biopsy. Clin Endosc 2018;51:181-185.

12. Tatsumi C, Kudo M, Ueshima K, et al. Non-invasive evaluation of hepatic fibrosis for type C chronic hepatitis. Intervirology 2010;53:76-81.

13. Wu J, You J, Yerian L, Shiba A, Schauer PR, Sessler DI. Prevalence of liver steatosis and fibrosis and the diagnostic accuracy of ultrasound in bariatric surgery patients. Obes Surg 2012;22:240-247.

14. Ryan CK, Johnson LA, Germin BI, Marcos A. One hundred consecutive hepatic biopsies in the workup of living donors for right lobe liver transplantation. Liver Transpl 2002;8:1114-1122.

15. Matteoni CA, Younossi ZM, Gramlich T, Boparai N, Liu YC, McCullough AJ. Nonalcoholic fatty liver disease: a spectrum of clinical and pathological severity. Gastroenterology 1999;116:1413-1419.

16. Jirapinyo P, Thompson CC. Sedation challenges: obesity and sleep apnea. Gastrointest Endosc Clin N Am 2016;26:527-537. 